\title{
Design of the calibration unit for the WEAVE multi-object spectrograph at the WHT
}

\author{
Lilian Domínguez ${ }^{1}$, Diego Cano ${ }^{1}$, Neil O’Mahony ${ }^{1}$, Carlos Martín ${ }^{1}$, Sergio Picó ${ }^{1}$, Chris Benn ${ }^{1}$, Scott \\ Trager $^{2}$, Emilie Lhomé ${ }^{1}$, Andy Ridings ${ }^{1}$, Cecilia Fariña ${ }^{1}$, D. C Abrams ${ }^{1}$, Marc Balcells ${ }^{1}$, Mike

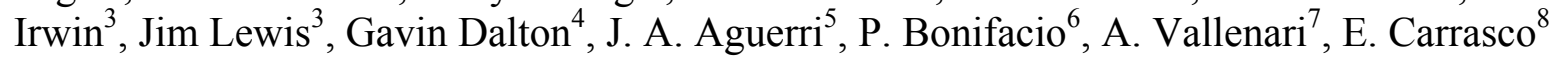 \\ ${ }^{1}$ Isaac Newton Group (ING); ${ }^{2}$ University of Groningen; ${ }^{3}$ Institute of Astronomy, Cambridge \\ University; ${ }^{4}$ STFC (RAL); ${ }^{5}$ Instituto de Astrofísica de Canarias (IAC); ${ }^{6}$ GEPI (Observatoire de \\ Paris); ${ }^{7}$ INAF; ${ }^{8}$ INAOE
}

\begin{abstract}
WEAVE is the next-generation spectroscopic facility for the William Herschel Telescope (WHT), offering multi-object (1000 fibres) and integral-field spectroscopy at two resolutions $(\mathrm{R} \sim 5000,20000)$ over a 2-deg field of view at prime focus. WEAVE will (mainly) provide optical follow up of ground-based (LOFAR) and space-based (GAIA) surveys. First light is expected in mid 2018.

Here, we describe the calibration unit, which will be adapted from an existing unit for the AF2+WYFFOS spectrograph (WEAVE's precursor) at the WHT. We summarise the results from a thorough characterisation of current performance (e.g. intensity, stability and focal-plane coverage of illumination as a function of lamp type and wavelength). We then set out our plans for upgrading the unit and its control systems to meet the WEAVE science and operational requirements.
\end{abstract}

We conclude from this assessment that the upgraded AF2+WYFFOS calibration unit will meet the requirements for WEAVE. The design of the WEAVE calibration unit is now complete.

Keywords: calibration unit, arc lamp, continuum source, multi-object, PAC, focal plane.

\section{INTRODUCTION}

WEAVE is a new wide-field spectroscopy facility proposed for the prime focus of the $4.2 \mathrm{~m}$ William Herschel Telescope (WHT). The facility comprises a new 2-degree field of view prime focus corrector with a 1000-multiplex fibre positioner, up to 20 individually deployable $11 \times 12 \operatorname{arcsec}^{2}$ integral field units, and a large single $78 \times 90 \operatorname{arcsec}^{2}$ integral field unit. The MOS and the IFUs fibres can be used to feed a dual-beam spectrograph that will provide full coverage of the majority of the visible spectrum $(3660-9590 \AA)$ in a single exposure at a spectral resolution of $\sim 5000$ or modest wavelength coverage $(4040-6850 \AA$ ) in both arms at a resolution 20000 (see WEAVE spectroscopic modes in Table 1, column 1). The instrument is expected to be on-sky by 2018 to provide spectroscopic sampling of the fainter end of the Gaia astrometric catalogue, chemical labelling of stars to $\mathrm{V} \sim 17$, and dedicated follow up of substantial numbers of sources from the medium deep LOFAR surveys.

At the current stage of the project, with the exception of the Prime Focus Corrector, all WEAVE products are being manufactured to final design. The WEAVE final design will ensure that the basic physical properties, for each system, are established and documented to a level that demonstrates the feasibility of the system design as well as its compliance with the overall architectural design and with the requirements. 
In this paper, we focus on the WEAVE calibration unit, whose design is based on the existing hardware installed at the WHT, currently in use for the AF2+WYFFOS multi-fibre spectrograph. The current calibration unit is being adapted to meet the science and operational requirements for WEAVE.

The focal-plane calibration unit allows the use of a series of lamps, with known spectrum, which are used to illuminate the field-of-view at prime focus via the tertiary mirror (M3) to feed light into the WEAVE fibres placed on the focal plane, provided the fibre positioner is mounted at prime focus. A schematic diagram of the calibration unit is given in Figure 1.

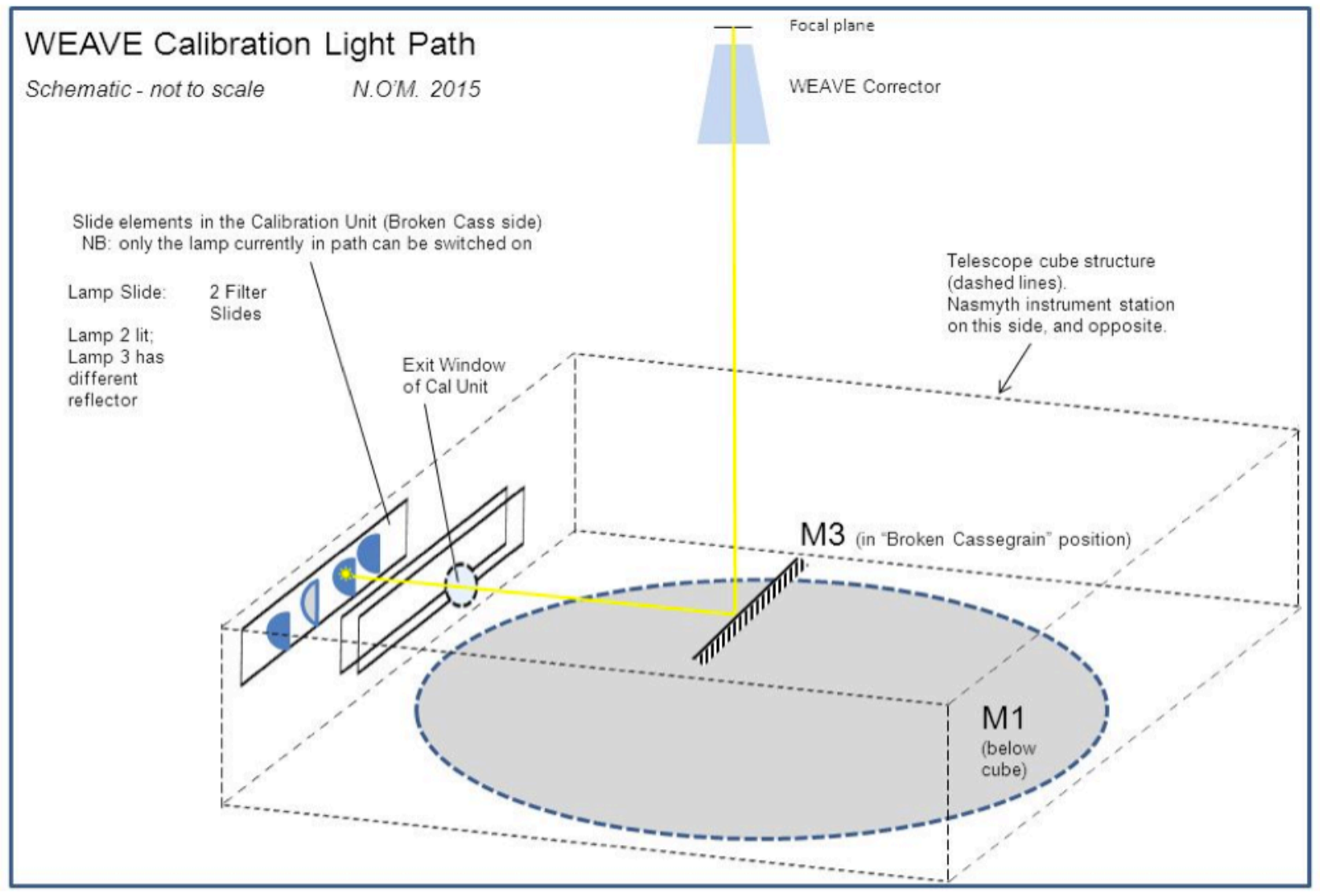

Figure 1. Diagram showing the light path from the lamps inside the WEAVE calibration unit to the focal plane where the WEAVE fibres are placed.

Lamp calibration exposures are required to calibrate the science data obtained each night, and also for long-term monitoring of the instrument stability.

The main types of calibrations exposures provided by the calibration unit are fibre flat, and arc frames. The fibre flats are normally used in multi-object spectrographs (MOS) to trace the location of each fibre on the detectors and its spatial profile. The fibres are illuminated by a continuum source, typically a quartz-tungsten-halogen (QTH) lamp, and produce fibre 'tram maps' on the detector, which are then used to define the apertures for summed (or optimal) extractions of each spectrum.

The arc lamps are used to illuminate the fibres for wavelength-calibration exposures. The arc lamps currently available for AF2+WYFFOS are Thorium-Argon (ThAr), Helium (He), Neon (Ne), Mercury (Hg), Cadmium (Cd) and Zinc (Zn). These lamps have well-known emission-line spectra, and arc-lamp exposures can be used to calibrate the wavelength scale for the science exposures. 


\section{ACHIEVEMENTS OF REQUIREMENTS}

The design of the focal-plane calibration module is based on existing mechanisms installed in the WHT for use with the AF2+WYFFOS spectrograph.

A complete assessment of the final design has been carried out at the ING to check that it meets the WEAVE science requirements. This assessment has included detailed measurements of light intensity using AF2+WYFFOS, and also using a wide-dynamic-range light meter in the focal plane of the telescope.

The requirements of the calibration unit can be grouped in the following categories:

- $\quad$ Requirements related to infrastructure (Section 3).

- Requirements related to control system (Section 4).

- $\quad$ Requirements related to speed and performance of mechanisms (Section 5).

- $\quad$ Requirements related to optical line coverage (Section 6).

- $\quad$ Requirements related to focal plane illumination (Section 7).

- $\quad$ Requirements related to repeatability of light intensity (Section 8).

In the following sections we provide details of the assessment we performed for each type of requirement and indicate whether the requirements are met.

\section{INFRASTRUCTURE}

The existing calibration unit consists of a 4-position slide holding calibration lamps, and two additional 6-position slides holding neutral density and coloured filters. Each of these slides is composed of a lead screw with a stepper motor and sensor switches that provide position feedback.

There are four parabolic reflectors in the lamp slide. One is expected to be permanently occupied by a QTH lamp, for the other three there is a choice of different lamps: $\mathrm{Hg}$, Ne and He discharge tube lamps and a hollow cathode ThAr lamp with a parabolic reflector matched to the lamp geometry. Recently $\mathrm{Zn}$ and $\mathrm{Cd}$ lamps have been used. Hence, three different arc lamps plus a continuum source will be available during the night for calibration purposes.

These lamps can be installed during the day by the ING engineering team, but should not be changed during the night as they are not easily accessible.

The filter slides will contain neutral density filters of different densities (at least ND =0.5, 1, 2, 3), and several colour filters, e.g. to attenuate the red spectral range relative to the blue for WEAVE low resolution mode.

A dark filter which, when positioned in front of the calibration-unit hole, prevents lamp flux to spill out will be also available in the filter slide, which is useful to allow lamps to warm up.

The calibration unit can be used for calibrations at any time, and at any telescope position, by pointing the tertiary mirror towards the "broken Cassegrain" aperture (where the lamps are located), which does not interfere with WEAVE observations (see Figures 1 and 2).

Since the proposed solution uses hardware already installed and working in the WHT, no difficulties related to the hardware integration are expected. The implementation design fulfils all the WEAVE requirements to integrate at the WHT. 


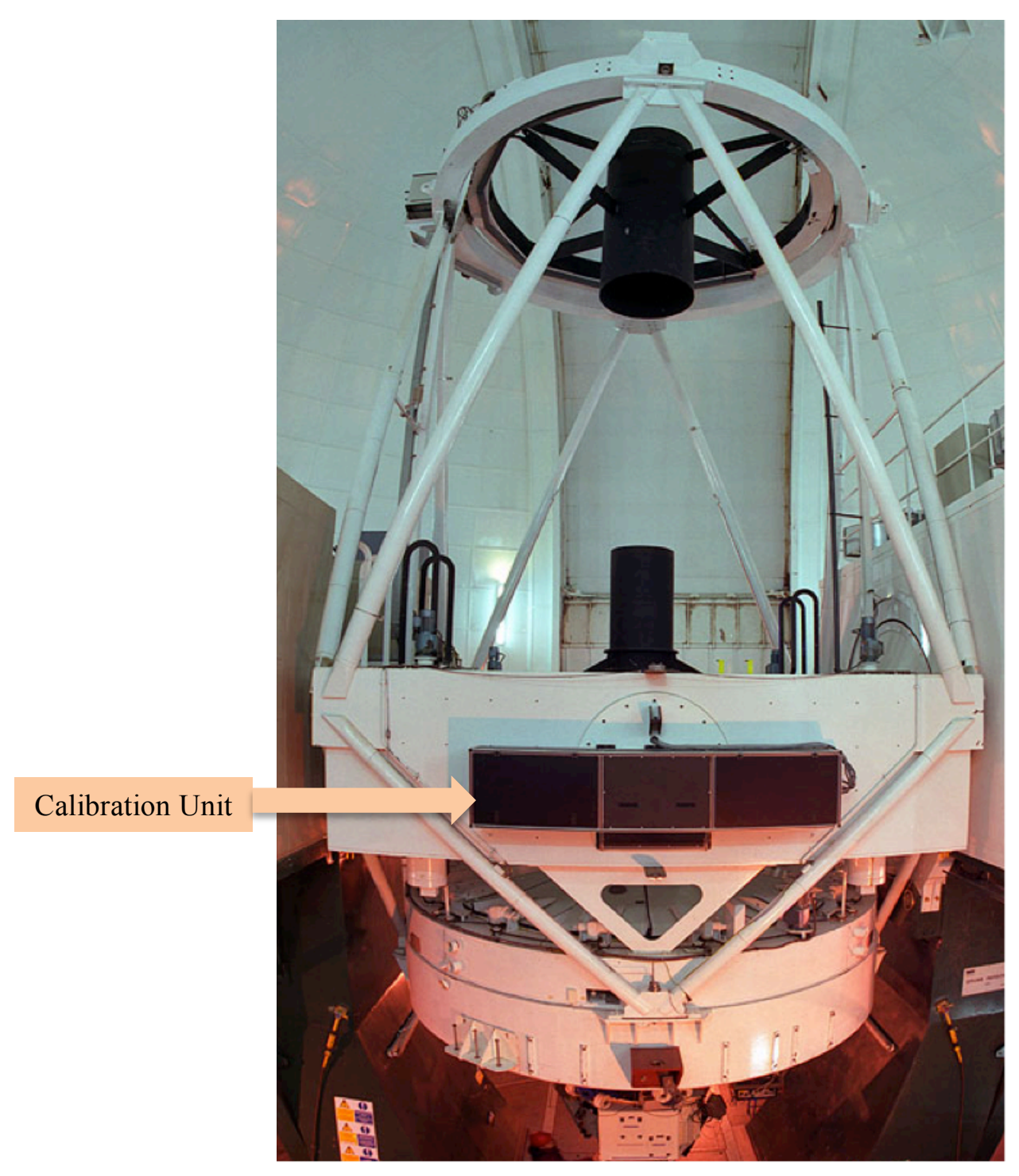

Figure 2. Calibration unit at William Herschel Telescope.

\section{NEW CONTROL SYSTEM}

Control of the calibration-unit mechanisms will be integrated into the WEAVE spectrograph's Programmable Automation Controller (PAC).

The proposed solution, already successfully tested in the laboratory, is to have a remote sub-controller (an Allen Bradley 1734 Point I/O, see Figure 3) that integrates all calibration-unit mechanisms and support equipment (encoders, sensors, limit switches, etc.) with the spectrograph PAC as a single controller. The remote controller will be connected via Ethernet to the spectrograph's PAC, so it will be acting as a slave and it is not intended to work independently, though it has a separate rack. Although physically separated, they are virtually the same unit from the controller programming 
point of view. This simplifies the development work as well as the integration tests. This sub-controller system has a wide range of modules capable of controlling the existing motor drivers, actuators and sensors without extra hardware.

This also allows a tidier cabling scheme since all the cables from the calibration-unit mechanisms are connected to this sub-controller rack, and do not mix with spectrograph cables.

As it can be seen in Figure 4, the sub-controller module rack houses its own power supply, one Ethernet module, 3 stepper motor controller modules (one for each motor), 3 digital input modules, one digital output module to switch on/off the lamps power supplies, and enable/disable the motors driver boards, and 2 analogue inputs modules to measure the current in the lamps.

Communication between the low-level software and the Observatory Control System (OCS) is established using the standard PLCIO library, which allows OCS mapping of the PAC memory and reading and writing of data. In this way, the embedded PAC code does not assume responsibility for updating the upper layers. It is just responsible for updating the internal data structures that will be read by the higher-level software, both the OCS and/or the engineering interface (Figure 5).

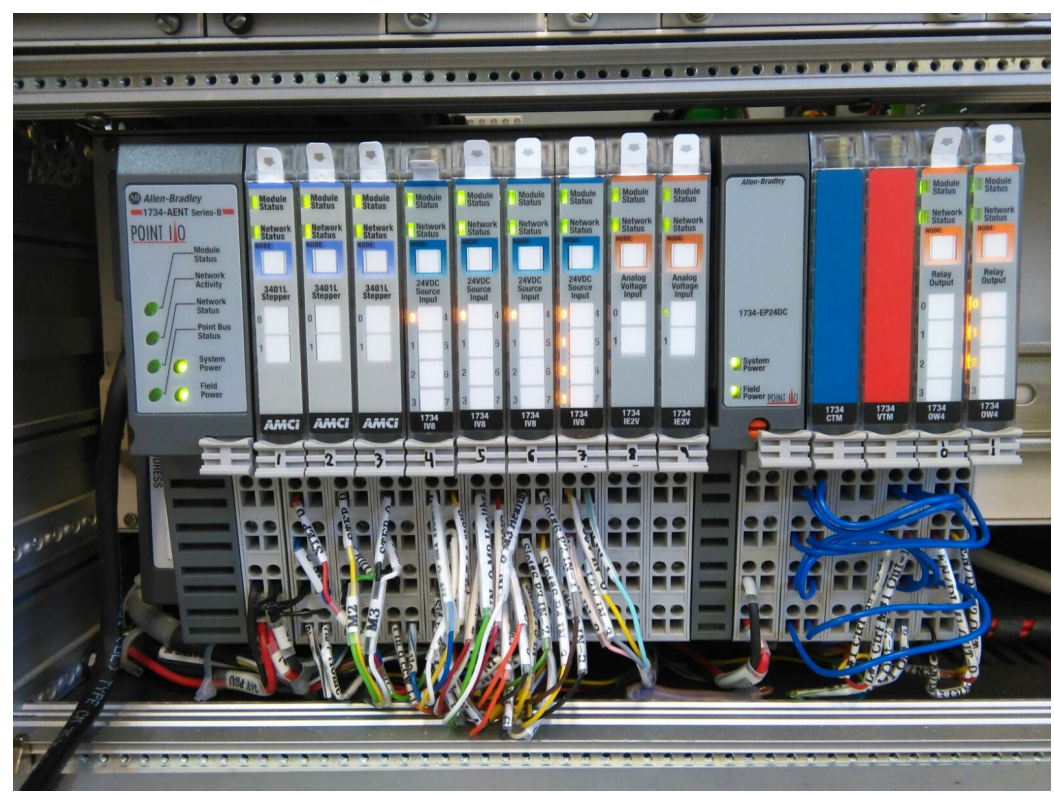

Figure 3. Allen Bradley 1734 Point I/O remote controller rack 


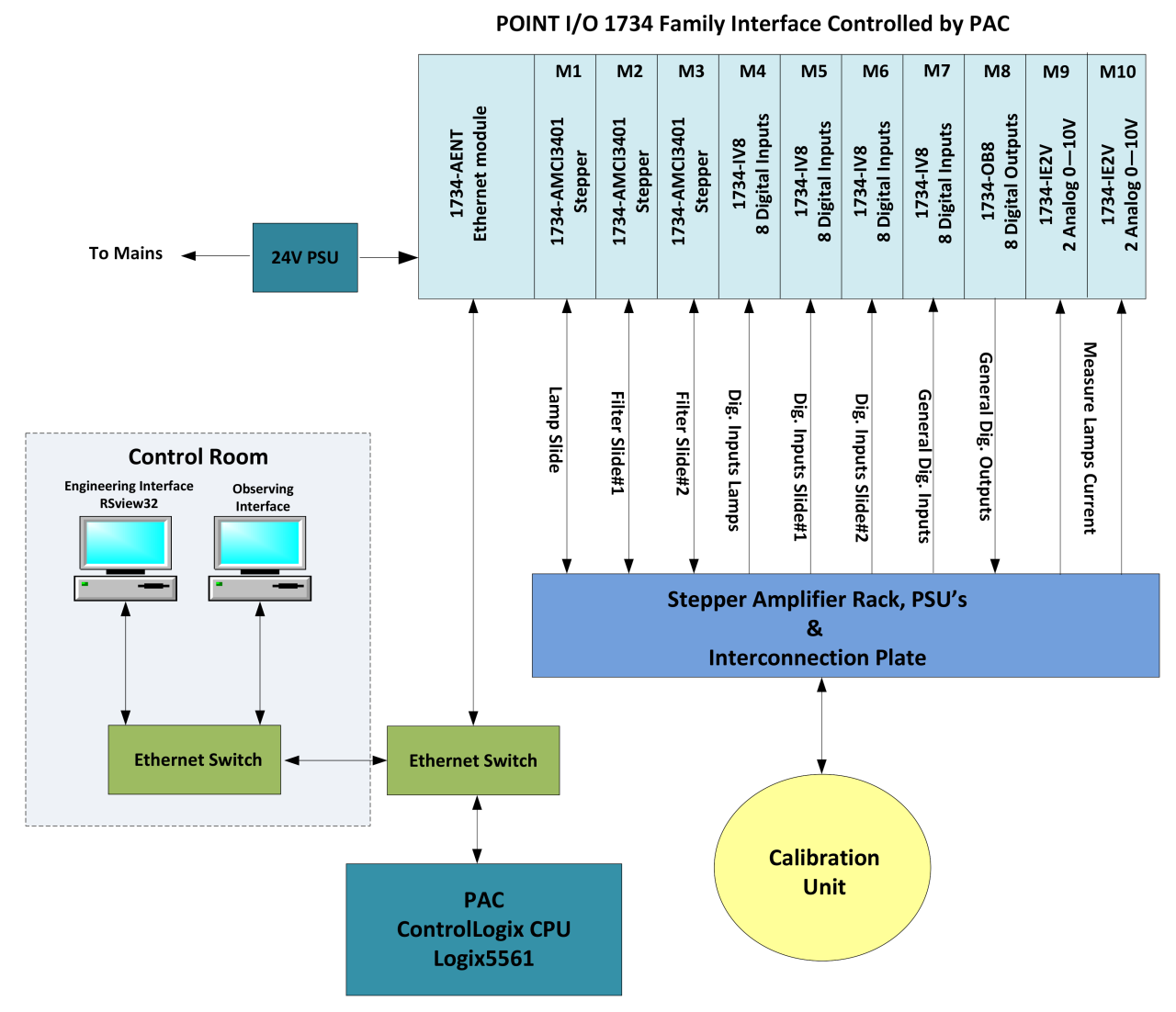

Figure 4. WEAVE calibration unit PAC control.

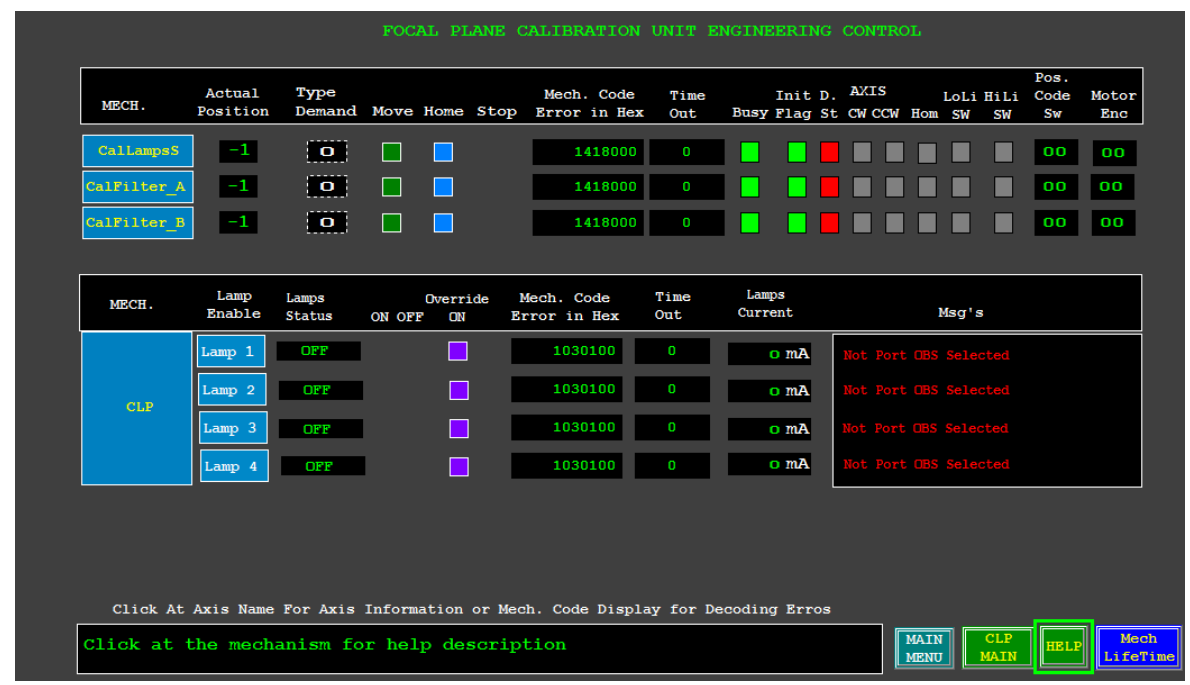

Figure 5. Engineering interface for the WEAVE calibration unit. 


\section{SPEED AND PERFORMANCE OF MECHANISMS}

By implementing a new control strategy for the stepper motor, we have increased the speed of calibration unit mechanisms by a factor of 5, without changing the hardware.

The stepper motors (Nanotec Electronic model SH8618M6008) have a step angle of $1.8^{\circ}$, i.e. 200 steps per revolution, with a maximum holding torque of $3.96 \mathrm{Nm}$ and quite low rotor inertia $\left(\mathrm{J}_{\text {motor }}=1.110^{-4} \mathrm{~kg} \mathrm{~m}^{2}\right)$, so the acceleration limits will be related to the inertia of the long lead screw (estimated $J_{\text {lead }}=7.510^{-4} \mathrm{~kg} \mathrm{~m}^{2}$ ) and the mass of lamps/filters block (estimated $20 \mathrm{~kg}$ ). Considering that the pitch of the lead screw is $0.5 \mathrm{~cm} / \mathrm{rev}$, the inertia contribution of the block mass is estimated $\mathrm{J}_{\text {block }}=1.210^{-5} \mathrm{~kg} \mathrm{~m}^{2}$ which is the smallest contribution to the system inertia. So the expected maximum angular acceleration achievable is:

$$
\alpha=T / J
$$

With $\mathrm{J}=\mathrm{J}_{\text {motor }}+\mathrm{J}_{\text {lead }}+\mathrm{J}_{\text {block}}$, and $\mathrm{T}=\mathrm{T}_{\max }-$ Friction. Estimating maximum torque $\left(\mathrm{T}_{\max }\right)$ at the speed of interest to be $50 \%$ of the maximum holding torque and the friction of $1 \mathrm{Nm}$, gives $\alpha$ of about $178 \mathrm{rev} / \mathrm{s}^{2}$, equivalent to a maximum linear acceleration of $0.89 \mathrm{~m} / \mathrm{s}^{2}$ of the slide.

To estimate the maximum speed achievable we must consider the characteristics of the motors winding (resistance $\mathrm{R}=$ $0.2 \Omega$, and inductance $\mathrm{L}=1.5 \mathrm{mH})$, the maximum current $\left(\mathrm{I}_{\max }=5 \mathrm{~A}\right)$, and the supply voltage $(\mathrm{V}=60 \mathrm{~V})$ of the driver module (McLennan Ltd. type PM546) with the stepper motor power supply (McLennan Ltd. type SWS1000L-60). Hence, the time constant of the motor coil is $\tau=\mathrm{L} / \mathrm{R}$ is $7.5 \mathrm{~ms}$, and the required voltage is $\mathrm{R} \times \operatorname{Imax}=1.7 \mathrm{~V}$, so at first approximation the minimum time per step is $\tau \times(\mathrm{R} \times \operatorname{Imax} / \mathrm{V})=0.12 \mathrm{~ms}$, so the expected achievable speed is about $8000 \mathrm{steps} / \mathrm{s}$, i.e. $40 \mathrm{rev} / \mathrm{sec}$, corresponding to a linear speed of $0.20 \mathrm{~m} / \mathrm{s}$ maximum. If maximum current is not requested to compensate the friction, even higher speeds could be achieved.

With all this in mind the expected maximum time for any slide movement is estimated to be below 20 seconds, with one second for ramp up and ramp down of the motor speed.

Whenever several exposures have to be taken with different lamps, generally the most efficient approach is to select the lamps sequentially, since the estimated swapping time is then about 5 seconds. Preliminary laboratory tests (with stepper motors similar to the ones in the unit and actual movement of the slides in the unit) have shown that the slide can be moved between extreme positions in about 10 seconds, meeting the speed requirement, which requested that the slides should be able to move from any position to any other in less than 30 seconds.

In Figure 6, we show the interconnection scheme for the lamp slide, and in Figure 7 the view of the test setup made in laboratory of the remote rack (Allen Bradley 1734 Point I/O) + PAC + dummy motors. 
WHT Weave Calibration Unit PAC Control

Lamp Slide\#1 \& Lamps Control

1734 Point I/O Family

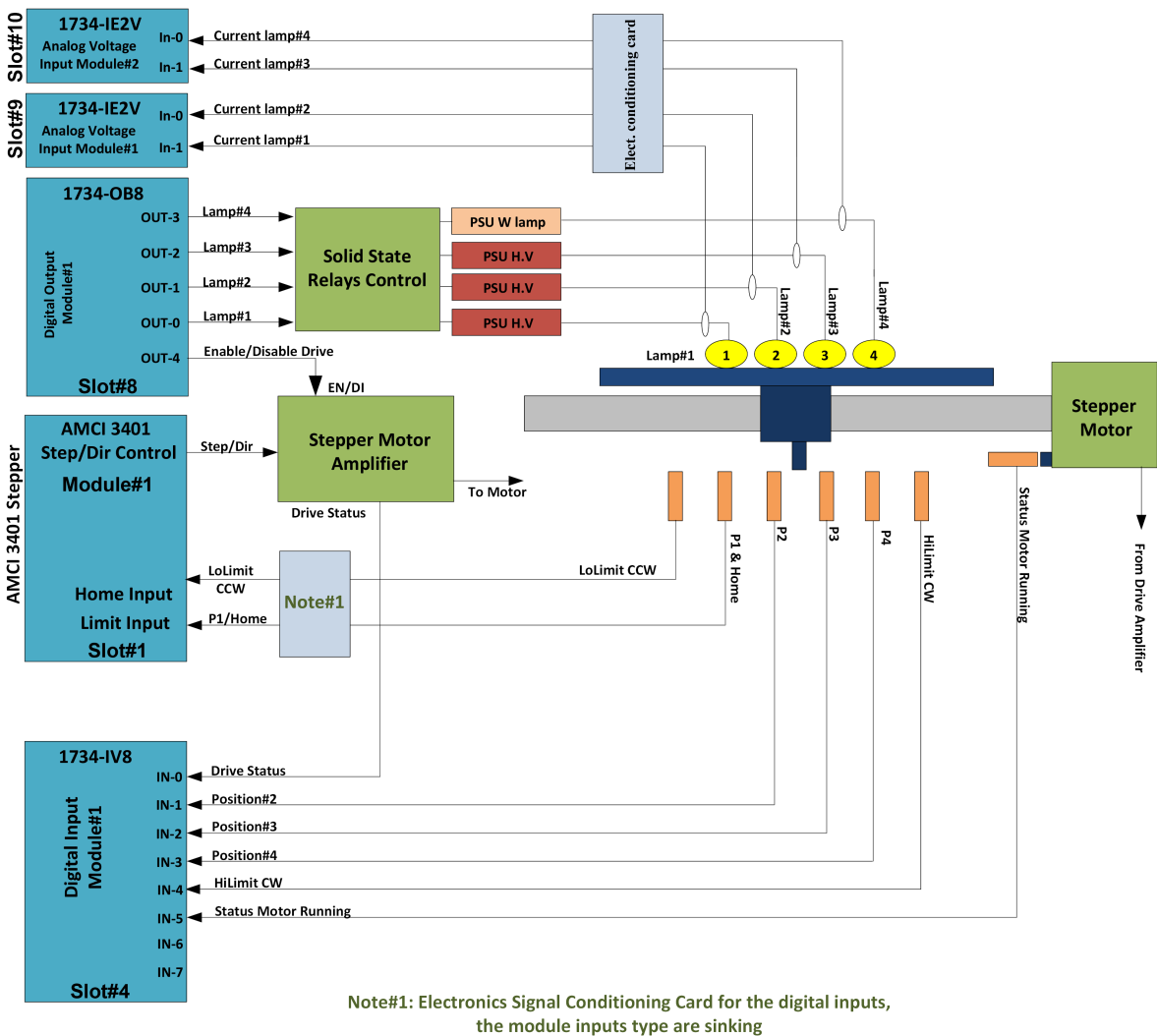

Figure 6. Interconnection scheme for the lamp slide.

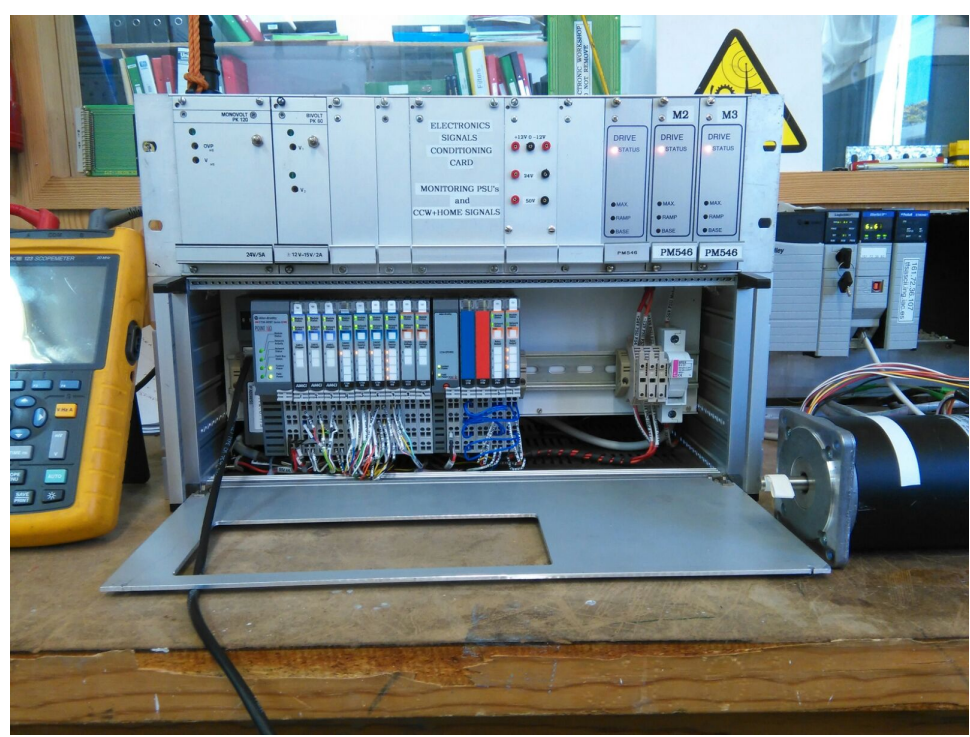

Figure 7. View of the remote rack (Allen Bradley 1734 Point I/O) + PAC + dummy motors: test setup in the laboratory. 


\section{WAVELENGTH COVERAGE}

The wavelength coverage of the current calibration-unit lamps (ThAr, $\mathrm{Ne}, \mathrm{He}, \mathrm{Hg}$ and $\mathrm{Cd}$ ) has been studied on the telescope using AF2+WYFFOS, to check if the science requirements are met for each configuration and spectral range of the WEAVE spectrograph. The requirements specify that the average number of lines must be greater than 10 lines/1000 $\AA$ for low resolution mode, and greater than 10 lines $/ 250 \AA$ for high resolution mode, corresponding to at least 30 spectral lines for each spectrograph arm.

Table 1 summarises the results from our measurements. The main conclusion is that the ThAr lamp is the most suitable lamp for WEAVE, meeting nearly all the requirements on arc-line density and coverage.

Table 1. Summary of lamp suitability for each WEAVE spectroscopic mode.

\begin{tabular}{|c|c|c|c|c|}
\hline WEAVE setup & $\begin{array}{c}\text { WEAVE } \\
\text { spectral range } \\
(\AA) \\
(2) \\
\end{array}$ & $\begin{array}{l}\text { Lamp option } 1 \\
\text { (matching the } \\
\text { requirements) } \\
\text { (3) }\end{array}$ & $\begin{array}{l}\text { Lamp option } 2 \\
\text { (matching or } \\
\text { almost matching } \\
\text { the requirements) } \\
\text { (4) }\end{array}$ & $\begin{array}{c}\text { Complementary } \\
\text { lamps } \\
\text { (far from matching } \\
\text { the requirements but } \\
\text { still useful) } \\
\text { (5) }\end{array}$ \\
\hline $\begin{array}{l}\text { Low-res } \\
\text { blue }\end{array}$ & $3660-6060$ & ThAr & --- & $\mathrm{Ne}, \mathrm{He}$ \\
\hline $\begin{array}{l}\text { Low-res } \\
\text { red }\end{array}$ & $5790-9590$ & ThAr & $\mathrm{Ne}$ & $\mathrm{Hg}, \mathrm{Cd}$ \\
\hline $\begin{array}{c}\text { High-res } \\
\text { blue } 1\end{array}$ & $4040-4650$ & ThAr & --- & --- \\
\hline $\begin{array}{c}\text { High-res } \\
\text { blue } 2\end{array}$ & $4730-5450$ & --- & ThAr & $\mathrm{Ne}$ \\
\hline $\begin{array}{l}\text { High-res } \\
\text { red }\end{array}$ & $5950-6850$ & ThAr & --- & $\mathrm{Ne}$ \\
\hline
\end{tabular}

For the low-resolution mode, the wavelength calibration is well covered, in both blue and red arms, by the ThAr lamp. In the red arm, the $\mathrm{Ne}$ lamp is also useful. The $\mathrm{He}, \mathrm{Hg}$ and $\mathrm{Cd}$ lamps may be useful complementary lamps, but do not fulfill the line-coverage requirement, with only a few arc lines in each WEAVE spectral range.

The ThAr also provides adequate calibration for the high-resolution red and blue arms, though it only nearly satisfies the requirements at high-resolution blue 2 mode.

Figure 8 shows an example of the spectrum of the ThAr lamp, and its arc line density in the spectral range $4300-4700$ $\AA$. The resolution of this spectrum is $\mathrm{R} \sim 18000$, close to the one for the WEAVE high-resolution mode. 


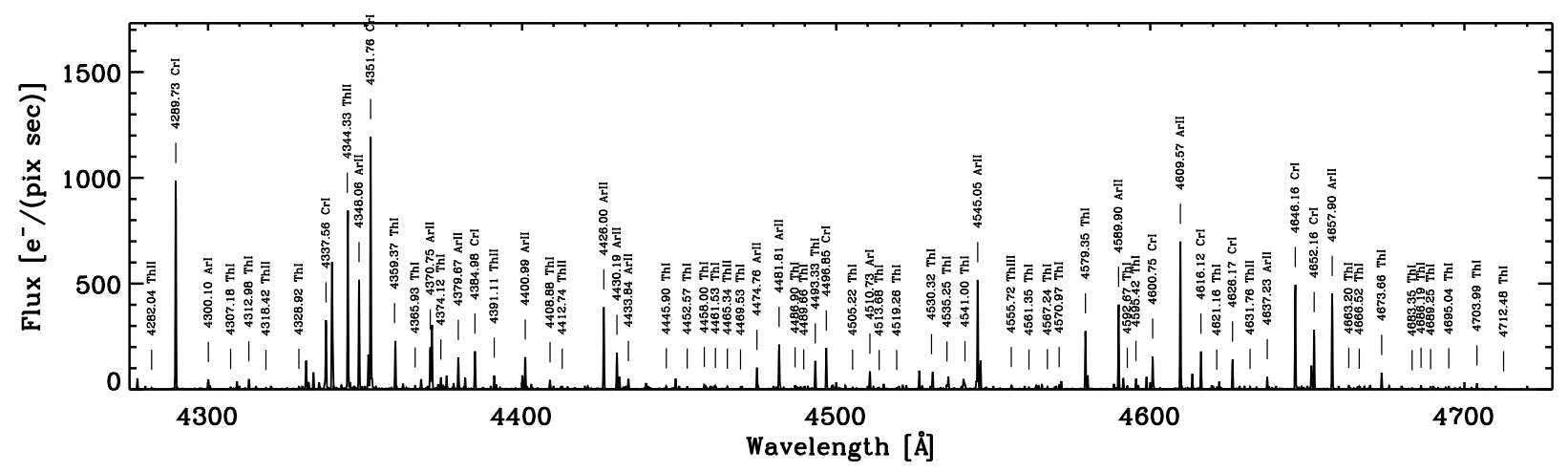

Figure 8. Example of arc map for the ThAr lamp $(\mathrm{R}=18000)$.

\section{FOCAL PLANE ILLUMINATION}

In order to measure the irradiance from the calibration lamps as a function of position in the focal plane, we produced a mechanical support for installation close to the focal plane. This mechanical support is a metal bar that can be positioned horizontally or vertically, passing through the optical axis. A specially-developed light-power meter, which has a low light level sensitivity and a wide dynamical range, can be positioned on this support, anywhere between the centre and the edge, thus sampling anywhere in the entire WEAVE field of view ( 2 degrees corresponding roughly to $\pm 20 \mathrm{~cm}$ from the centre).

The results for the QTH, Ne, and He lamps, show that the radial variation in illumination is less than $25 \%$ over the $40 \mathrm{~cm}$ diameter WEAVE field, fulfilling the requirement that specify that the radial variation shall be $<50 \%$.

In the case of the Mercury pen ray lamp ( $\mathrm{Hg})$, the irradiance decreases $35 \%$ at the edge of the measured area with respect to the brightest central part. Laboratory tests with this lamp showed that using a diffuser greatly improves the uniformity, although it reduces peak irradiance by approximately $50 \%$.

We evaluated the exposure times to get the required signal-to-noise ratio (SNR) on the arc lines for these lamps. Given the WEAVE science requirement for wavelength accuracy $<0.05$ pixels, and that the FWHM at the WEAVE CCDs will be typically $\sim 3$ pixels, the required SNR on the arc exposure is presumably $\sim(\mathrm{FWHM} /$ accuracy), i.e. SNR $\sim(3 / 0.05)$ $\sim 60$. This implies a total of $\sim 3600$ photons in each arc line (integrated, not peak) for each of the five spectral modes of WEAVE. Taking into account the ratio in throughput between AF2+WYFFOS and WEAVE, we estimate that for these lamps ( $\mathrm{He}, \mathrm{Ne}, \mathrm{Hg}$, and $\mathrm{Cd}$ ) we need exposures $<30$ seconds to get $\sim 3600$ photons in enough arc lines. This fulfils the WEAVE requirement that the calibration exposure times should be $<30$ seconds (optimally).

In the case of the ThAr hollow-cathode lamp, the principal issue is that it is intrinsically very faint, especially in the blue. The main implication from this is that the exposure times may be much longer than 30 seconds, up to 120 sec in the high-resolution blue 2 WEAVE mode. Additionally, the size and uniformity of the illumination depends on the position of the hollow-cathode with respect to the paraboloid focus, which is more difficult to adjust with a faint lamp.

To solve this issue, a new ThAr lamp from Photron Ltd. (Australia) has been purchased for WEAVE, which allows an excitation current 4 times higher. By a careful adjustment of the lamp position within the reflector, we have found that: 
- It improves the flux of the arc lines a factor 2-3 allowing shorter exposure times, close or below the optimal requirement of $30 \mathrm{sec}$.

- It illuminates the WEAVE field of view with the required uniformity. This is with a variation of relative illumination $<50 \%$ (see Figure 9)

- The reflector mount should undergo mechanical modifications to improve adjustability, stability and repeatability.

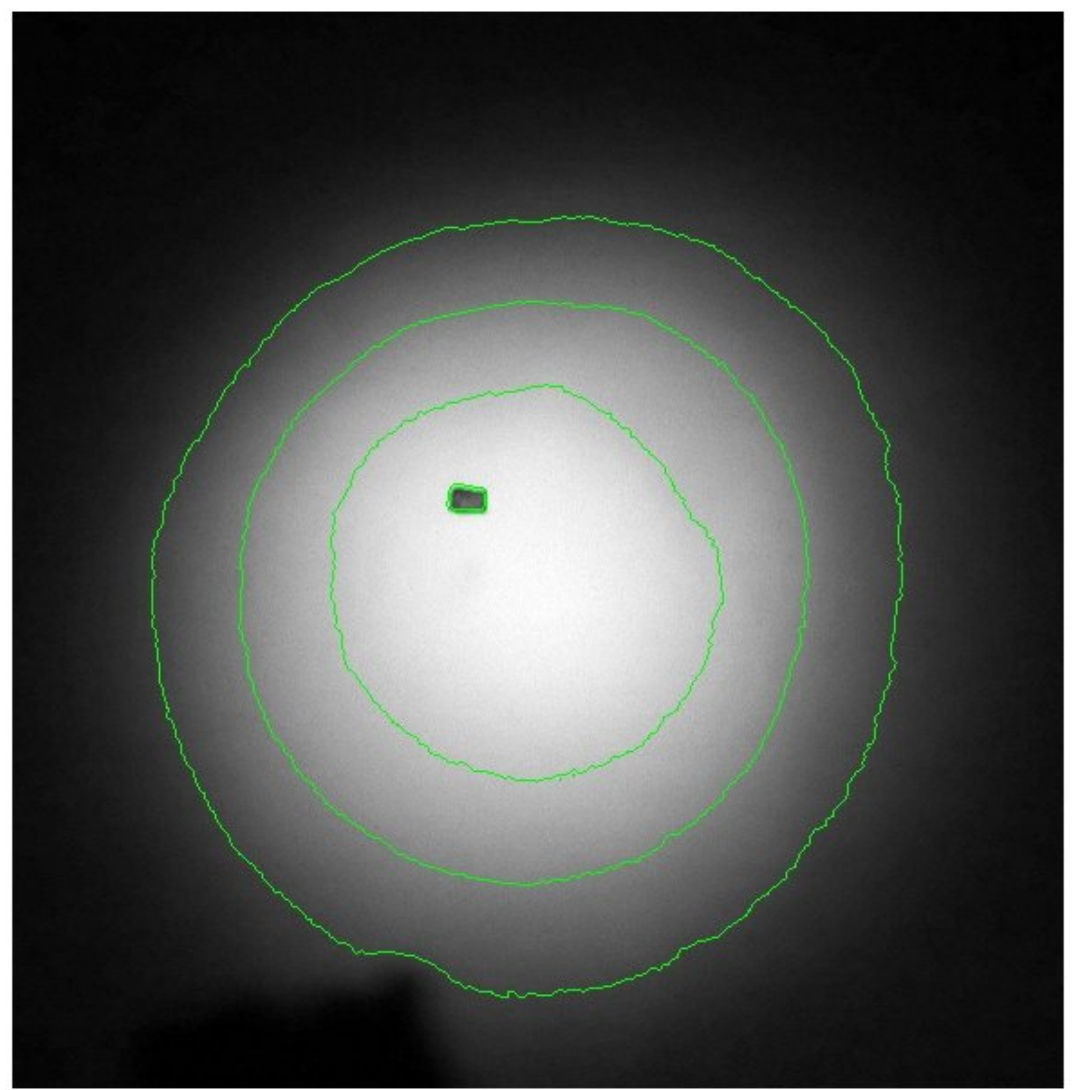

Figure 9. Beam of new ThAr lamp with optimal focus adjustment. The contours correspond to $75 \%, 50 \%$ and $25 \%$ of maximum, respectively. The inner black spot is an alignment target. The second contour (50\%) is at $\sim 37-38 \mathrm{~cm}$ diameter, which roughly match the diameter of the WEAVE field of view.

In Figure 10, we can see the new ThAr lamp mounted in a special reflector inside the calibration unit, between other two conventional lamp reflectors. 


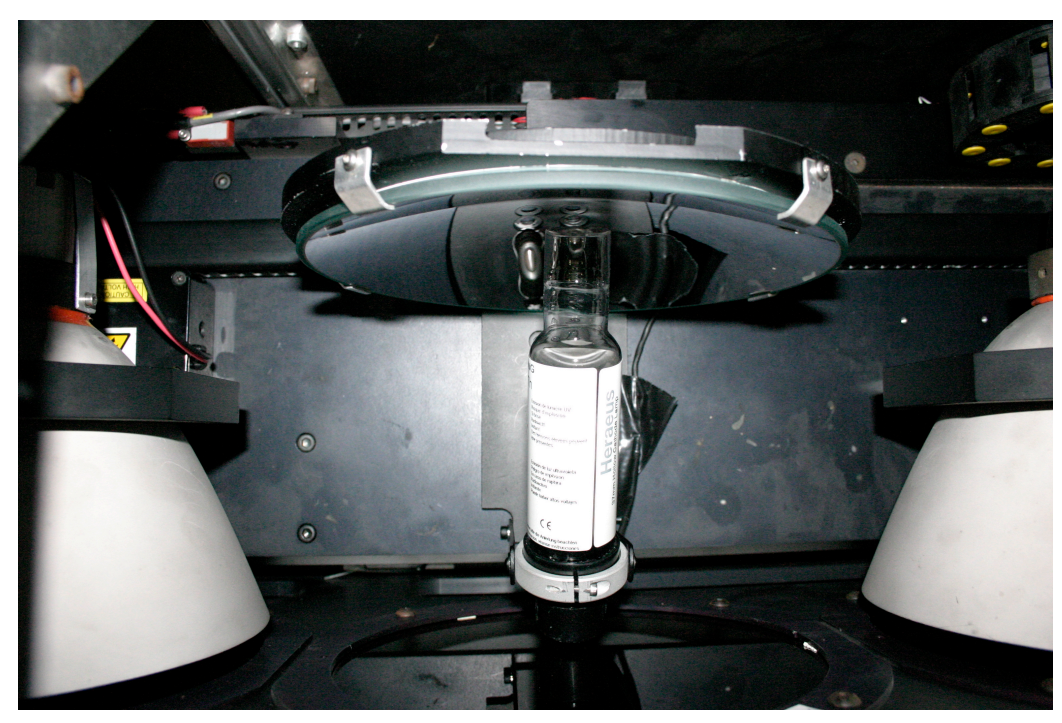

Figure 10. New ThAr lamp and special reflector installed in the calibration unit between other two conventional lamp reflectors.

\section{REPEATABILITY OF LAMP INTENSITY}

The variation of lamp intensity with time was measured for all the available lamps, including lamp response times, brightness variations, warm-up times, and long-term stabilization. Both integrated lamp flux and relative/total arc-line variation were analysed in detail.

The results of these investigations are that, following warm up, all of the spectral lamps display small variations $(<10 \%)$ in relative line intensity occurring over periods of time typically longer (200 - 1200 seconds) than the typical calibration exposure times ( 2 to 120 seconds). Hence, successive exposures can be made with highly repeatable signal.

The largest variation in intensity happens typically in the first 30 seconds after switching on, particularly for the Hg lamp (see Figure 11). In this case, it is accompanied by a significant change in the distribution of spectral intensities during this time. This is probably due to the increasing dominance of Mercury lines over those of the fill gas Argon, as the liquid metal is vaporised. Something similar happens with the Cd lamp, which also contains Argon. These lamps need from 200 to $300 \mathrm{sec}$ to stabilise.

After the initial warm-up, the lamps typically exhibit a $10-20 \%$ rise in total intensity over periods of 900 to 1200 seconds.

The ThAr and Ne lamps show practically no need for warm-up (see Figure 12), although it may be beneficial to warm the ThAr lamp for a few seconds ( $<60$ seconds) when observing in the blue to allow faint lines in there to become brighter. 


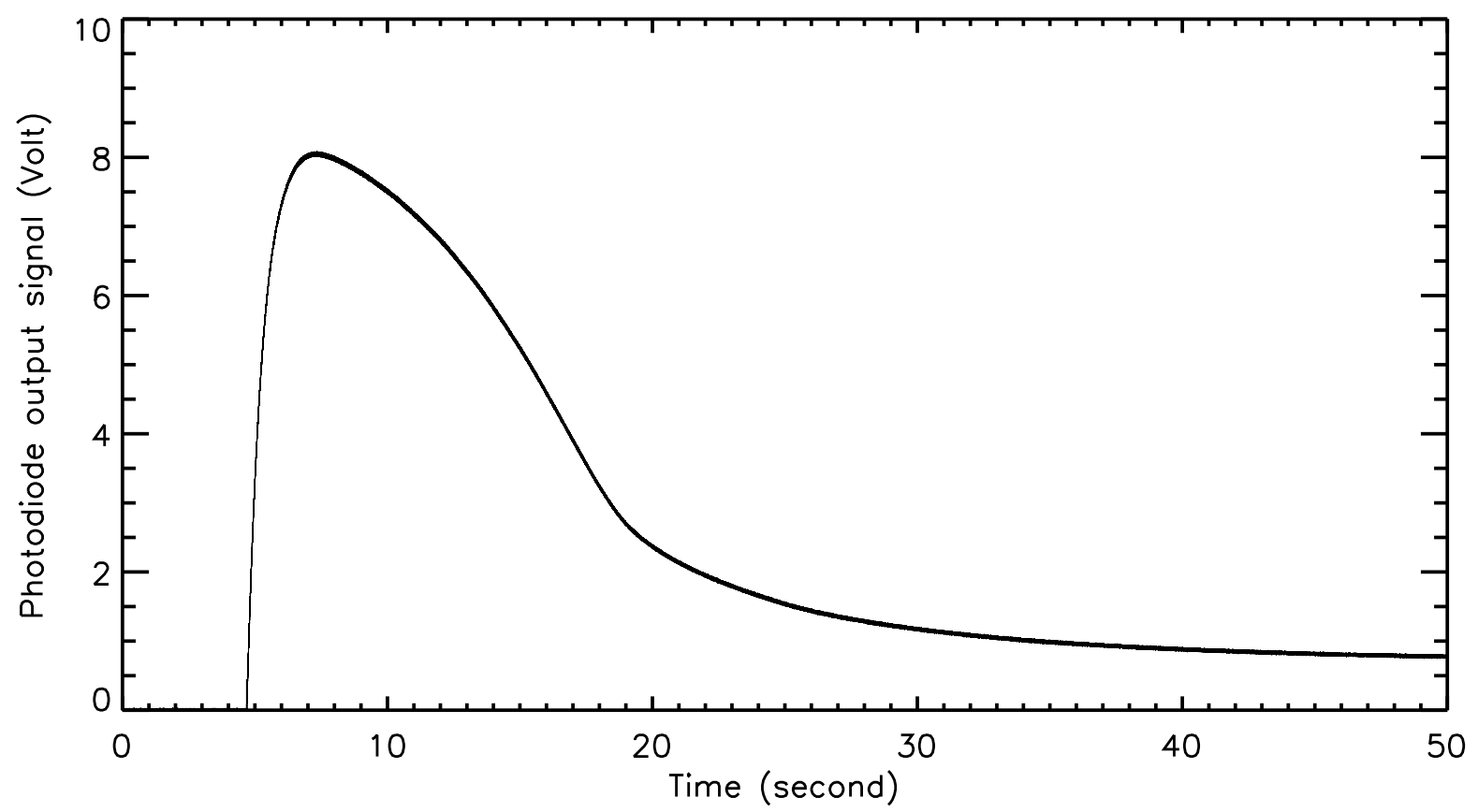

Figure 11. Power-up response of the Hg lamp with output signal from the photodiode (Volts, in arbitrary voltage scale) versus time (seconds).

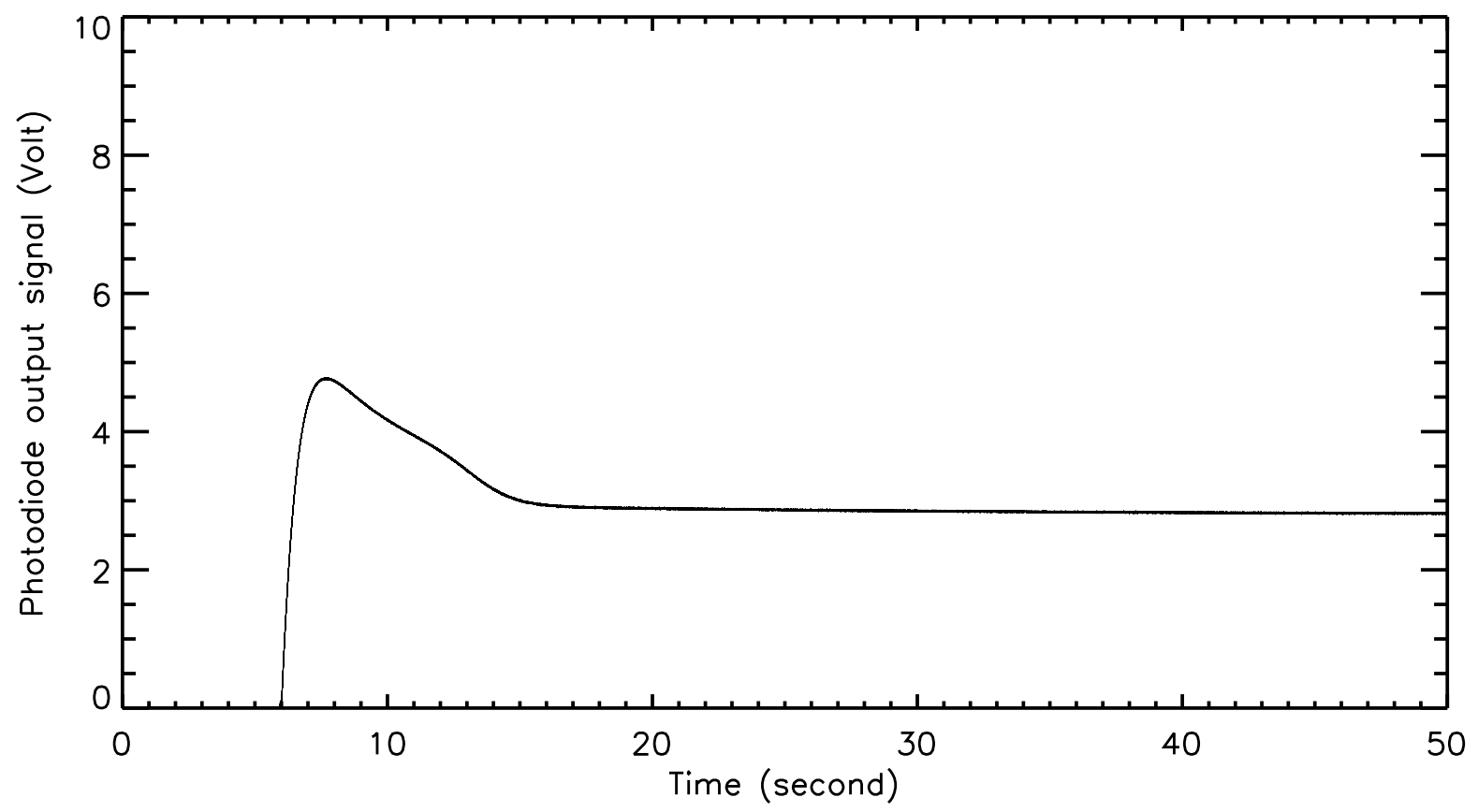

Figure 12. Power-up response of the ThAr lamp with output signal from the photodiode (Volts, in arbitrary voltage scale) versus time (seconds). 


\section{CONCLUSIONS}

The design of the WEAVE calibration module is now complete. It is based on the existing mechanisms currently installed at the WHT for use with AF2+WYFFOS. An exhaustive assessment of the current performance of the unit has been carried out, and we conclude that after some upgrades (new control system, new control strategy, new lamps) the module should meet the WEAVE science and operational requirements.

\section{REFERENCES}

[1] Dalton, Gavin B et al. "Final design and progress of WEAVE: the next generation wide-field spectroscopy facility for the William Herschel Telescope", Proc. SPIE AS16-AS105-22 (2016). 